\title{
AN APPROXIMATE ANALYSIS AND ITS APPLICATION TO THE NON-LINEAR PERFORMANCE OF THREE MOSFET TRANSCONDUCTANCE AMPLIFIERS
}

\author{
MUHAMMAD TAHER ABUELMA'ATTI \\ King Fahd University of Petroleum and Minerals, Box 203, Dhahran 31261, Saudi Arabia
}

(Received March 29, 1994; in final form May 11, 1994)

\begin{abstract}
A simple procedure for approximating the input-output characteristic of non-linear electronic circuits is presented. Using this procedure, closed-form analytical expressions, in terms of the ordinary Bessel functions, are obtained for the output spectra of a non-linear electronic circuit resulting from a multisinusoidal input. Using these expressions, the non-linear performance of three basic MOSFET transconductance amplifiers is considered in an attempt to determine the transistor parameters for best linearity.
\end{abstract}

\section{INTRODUCTION}

It is frequently valuable in the design of analog signal processing circuits and systems to have an indication of the likely spectrum of output signals, including harmonics and intermodulation products, from a non-linear component of the system whose characteristics are known only in the form of an untractable mathematical expression relating its input and output variables. A widely used procedure for evaluating output spectra involves derivation of a high-order polynomial approximation to the available untractable mathematical expression and this, in turn, implies the use of sophisticated curve-fitting techniques that invariably demand extensive computing facilities and well developed software. A problem, however, arises when the mathematical expression available represents the input variable as a function of the output variable, while what we would prefer is an expression for the output variable as a function of the input variable. This requires a series inversion to get the output variable as a function of the input variable. Moreover, for an nth-order polynomial, the harmonics and intermodulation products that can be evaluated are restricted to nth-order only.

An alternative procedure, which permits the derivation of high-order product magnitudes from a simple model must, therefore, meet the following two principle requirements: a. the mathematical description, which is valid over a wide range of the input variable, must be of a kind that incorporates the major features of the characteristics of the non-linear component while still offering straightforward means for calculating harmonic and intermodulation products magnitudes; and $\mathrm{b}$. 
having obtained such a mathematical model it must be possible to derive its parameters without recourse to the use of sophisticated curve-fitting techniques.

It is the major intention of this paper to present such a procedure. In principal, the proposed procedure is based on approximating the non-linear component characteristics by a sine-series function. The coefficients of this sine-series function can be obtained using simple hand calculations. To illustrate the effectiveness of the proposed procedure, approximations of the non-linear characteristics of three widely used MOSFET transconductance amplifiers will be considered. Evaluation of the harmonic distortion performance of these amplifiers will be also considered in an attempt to determine the transistor parameters for best linearity.

\section{PROPOSED APPROXIMATION}

In general, the input-output characteristic of a non-linear electronic component will be available in the form of a mathematical expression. The development of the present approximation proceeds along empirical lines by comparing the truncated sine-series model of (1) with the available mathematical expression of the input-output characteristic of the non-linear component under consideration.

$y=\sum_{n=1,3,5} \gamma_{n} \sin \left(\frac{n \pi}{B} x\right)$, for $\frac{-B}{2} \leqslant x \leqslant \frac{B}{2}$

where $x$ and $y$ are the input and output variables respectively. This implies representation of the output of the non-linear component by a sine-series in the input variable with periodicity, equal to $2 \mathrm{~B}$, chosen such that the working range of the input variable is an appropriate segment around zero. The parameters $\gamma_{n}, n=1$, 3,5 can be obtained using the twelve-point method [1]. In general, this procedure will result in a family of parameters $B$ and $\gamma_{n}$ that depend on the physical and dimensional properties of the component under consideration. The parameters $\mathrm{B}$ and $\gamma_{n}, n=1,3,5$ are then fitted to simple, closed-form analytical expressions of the form

$\gamma_{\mathrm{n}}=\sum_{\mathrm{m}=0}^{5} \delta_{\mathrm{nm}} \alpha^{\mathrm{m}}$

where $\alpha$ is the electronic-component parameter that depends on its physical and dimensional properties. Closed-form analytical expressions other than (2) can also be used wherever appropriate. Thus, by combining (1) and (2) the global approximation proposed here can be expressed as

$$
y=\sum_{n=1.3 .5} \sum_{m=0}^{5} \delta_{n m} \alpha^{m} \sin \left(\frac{n \pi}{B} x\right), \text { for } \frac{-B}{2} \leqslant x \leqslant \frac{B}{2}
$$




\section{HARMONIC AND INTERMODULATION ANALYSIS}

One of the intended applications of the proposed approximation of (1) is to predict the non-linear performance of an electronic component excited by a multisinusoidal input signal of the form

$\mathrm{x}=\sum_{\mathrm{k}=1}^{\mathrm{K}} \mathrm{X}_{\mathrm{k}} \sin \omega_{\mathrm{k}} \mathrm{t}$

where $\Sigma\left|\mathrm{X}_{\mathrm{k}}\right| \leqslant \mathrm{B} / 2$.

Substituting (4) into (1) yields

$\mathrm{y}=\sum_{\mathrm{n}=1.3 .5} \gamma_{\mathrm{n}} \sin \left(\frac{\mathrm{n} \pi}{\mathrm{B}} \sum_{\mathrm{k}=1}^{\mathrm{K}} \mathrm{X}_{\mathrm{k}} \sin \omega_{\mathrm{k}} \mathrm{t}\right)$

Using the identities

$$
\begin{aligned}
& \sin (\mathrm{z} \sin \phi)=2 \sum_{\mathrm{l}=0}^{\infty} \mathrm{J}_{2 \mathrm{l}+1}(\mathrm{z}) \sin (2 \mathrm{l}+1) \phi \\
& \cos (\mathrm{z} \sin \phi)=\mathrm{J}_{\mathrm{o}}(\mathrm{z})+2 \sum_{\mathrm{l}=1}^{\infty} \mathrm{J}_{2 \mathrm{l}}(\mathrm{z}) \cos 2 \mathrm{l} \phi
\end{aligned}
$$

it is easy to show that the amplitude of an output component of frequency

$\sum_{k=1}^{K} \lambda_{k} \omega_{k}$

where $\lambda_{\mathrm{k}}$ is a positive or negative integer or zero, will be given by

$$
\mathrm{Y}_{\lambda_{1}, \lambda_{2}, \ldots, \lambda_{\mathrm{K}}}=2 \sum_{\mathrm{n}=1,3,5} \gamma_{\mathrm{n}} \prod_{\mathrm{k}=1}^{\mathrm{K}} \mathbf{J}_{\left|\lambda_{\mathrm{k}}\right|}\left(\frac{\mathrm{n} \pi}{\mathrm{B}} \mathrm{X}_{\mathrm{k}}\right)
$$

where $\mathbf{J}_{\left|\lambda_{k}\right|}($.$) is the Bessel function of the order \left|\lambda_{k}\right|$ and $\Sigma_{k=1}^{K}\left|\lambda_{k}\right|$ is the order of the output product and is an odd integer. Therefore, the amplitude of an output product of frequency $\omega_{\mathrm{p}}$ is

$$
Y_{1}=2 \sum_{n=1,3,5} \gamma_{n} J_{1}\left(\frac{n \pi}{B} X_{p}\right) \prod_{\substack{k=1 \\ k \neq p}}^{K} J_{o}\left(\frac{n \pi}{B} X_{k}\right)
$$

the amplitude of a third-harmonic product of frequency $3 \omega_{\mathrm{p}}$ is

$$
Y_{3}=2 \sum_{n=1.3 .5} \gamma_{n} J_{3}\left(\frac{n \pi}{B} X_{p}\right) \prod_{\substack{k=1 \\ k \neq p}}^{K} J_{o}\left(\frac{n \pi}{B} X_{k}\right)
$$


and the amplitude of a fifth-harmonic product of frequency $5 \omega_{\mathrm{p}}$ is

$\mathrm{Y}_{5}=2 \sum_{\mathrm{n}=1.3 .5} \gamma_{\mathrm{n}} \mathrm{J}_{5}\left(\frac{\mathrm{n} \pi}{\mathrm{B}} \mathrm{X}_{\mathrm{p}}\right) \prod_{\substack{\mathrm{k}=1 \\ \mathrm{k} \neq \mathrm{p}}}^{\mathrm{K}} \mathrm{J}_{\mathrm{o}}\left(\frac{\mathrm{n} \pi}{\mathrm{B}} \mathrm{X}_{\mathrm{k}}\right)$

In a similar way, the amplitude of a harmonic or intermodulation product of any order can be obtained using (6).

\section{EXAMPLES}

In this section the harmonic performance of three widely used MOSFET transconductance amplifiers will be considered as illustrative examples for the use of the procedure presented in the previous sections.

\subsection{Example 1}

Consider the weak inversion MOSFET transconductance amplifier, shown in Fig. 1. Assuming that the two transistors are perfectly matched, the current-voltage characteristic of the transconductance amplifier of Fig. 1, with the MOSFETs work-

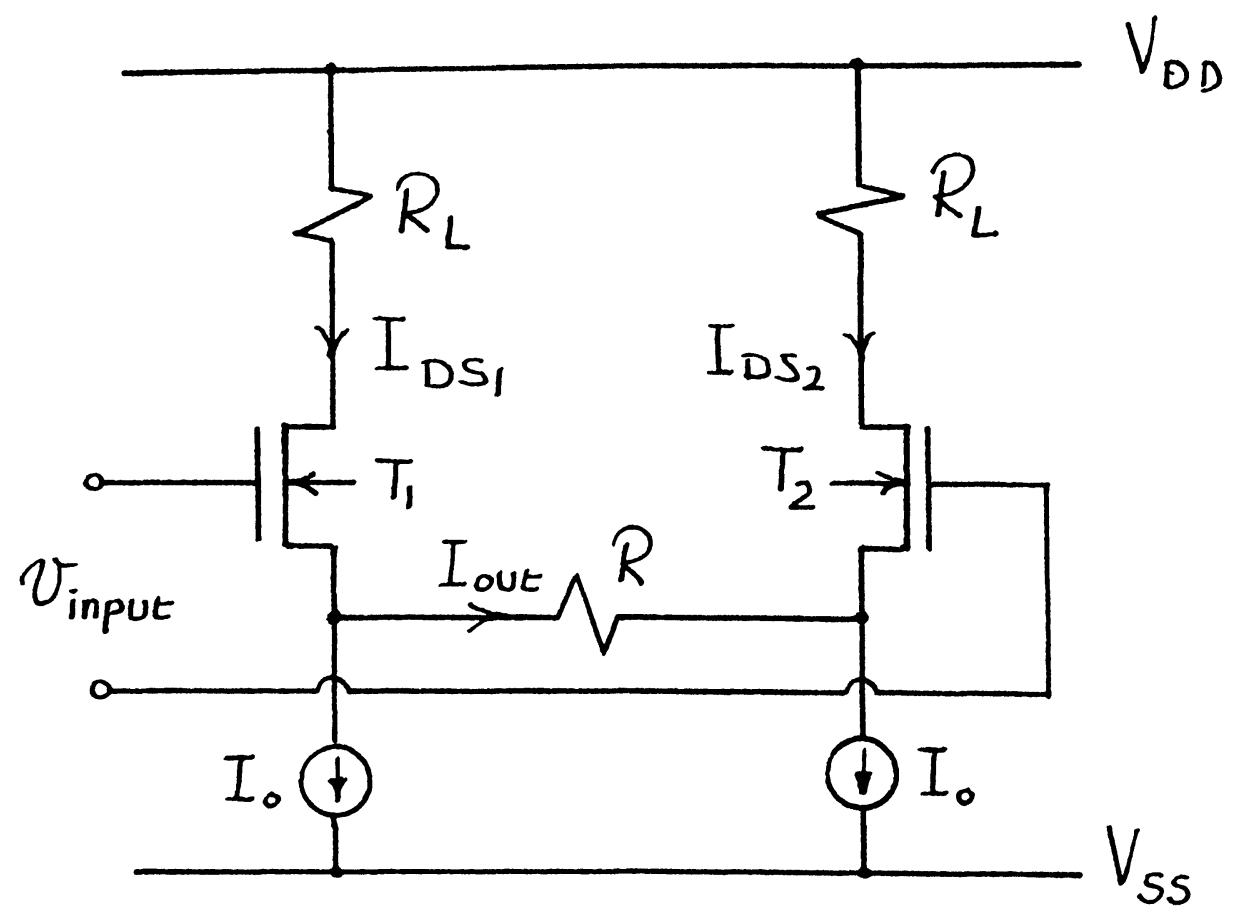

FIGURE 1 MOSFET transconductance amplifier 
ing in the subthreshold region, can be expressed as

$\nu_{\mathrm{D}}=\alpha \mathrm{i}_{\mathrm{out}}+\ln \left(\frac{1+\mathrm{i}_{\text {out }}}{1-\mathrm{i}_{\text {out }}}\right)$

where $\nu_{\mathrm{D}}=\nu_{\text {input }} / \mathrm{n} \theta, \mathrm{i}_{\text {out }}=\mathrm{I}_{\text {out }} / \mathrm{I}_{\mathrm{o}}, \alpha=\mathrm{I}_{\mathrm{o}} \mathrm{R} / \mathrm{n} \theta$ and the bias current $\mathrm{I}_{\mathrm{o}}$ should be less than $1 \mu \mathrm{A}$ to ensure that the MOSFETs are working in the subthreshold region.

Equation (10) in its present form cannot be used to investigate the non-linear performance of the weak inversion transconductance amplifier of Fig. 1, as this requires an expression for the output current as a function of the input voltage. Using the procedure described in the preceding sections, (10) can be approximated by the sine-series function of (1), with $\mathrm{x}=\nu_{\mathrm{D}}$ and $\mathrm{y}=\mathrm{i}_{\text {out }}$ for any value of $\alpha$. For different values of $\alpha$, the parameters $B$ and $\gamma_{n}, n=1,3,5$ were obtained, and fitted to (2), giving

$$
\begin{aligned}
& \gamma_{1}= \frac{1.1}{1+0.031 \alpha-0.0014 \alpha^{2}} \\
& \gamma_{3}=0.126-0.43\left(\frac{\alpha}{10}\right)+0.1458\left(\frac{\alpha}{10}\right)^{2}+0.7292\left(\frac{\alpha}{10}\right)^{3}-1.1458\left(\frac{\alpha}{10}\right)^{4}+0.5208\left(\frac{\alpha}{10}\right)^{5} \\
& \gamma_{5}=0.026-0.8786\left(\frac{\alpha}{10}\right)+6.2156\left(\frac{\alpha}{10}\right)^{2}-15.8599\left(\frac{\alpha}{10}\right)^{3} \\
&+16.8906\left(\frac{\alpha}{10}\right)^{4}-6.3880\left(\frac{\alpha}{10}\right)^{5}
\end{aligned}
$$

and

$\mathrm{B}=10.6+2 \alpha$

Using (1) and (11-14), calculations were made and the results are shown in Fig. 2. From Fig. 2, it is obvious that the proposed approximation accurately represents the input-output characteristic of the weak inversion MOSFET transconductance amplifier shown in Fig. 1.

Now, consider a weak inversion MOSFET differential amplifier excited by a linear time varying signal of the form

$$
\nu_{\mathrm{D}}=\frac{1}{2} \mathrm{Bt} / \mathrm{T}
$$




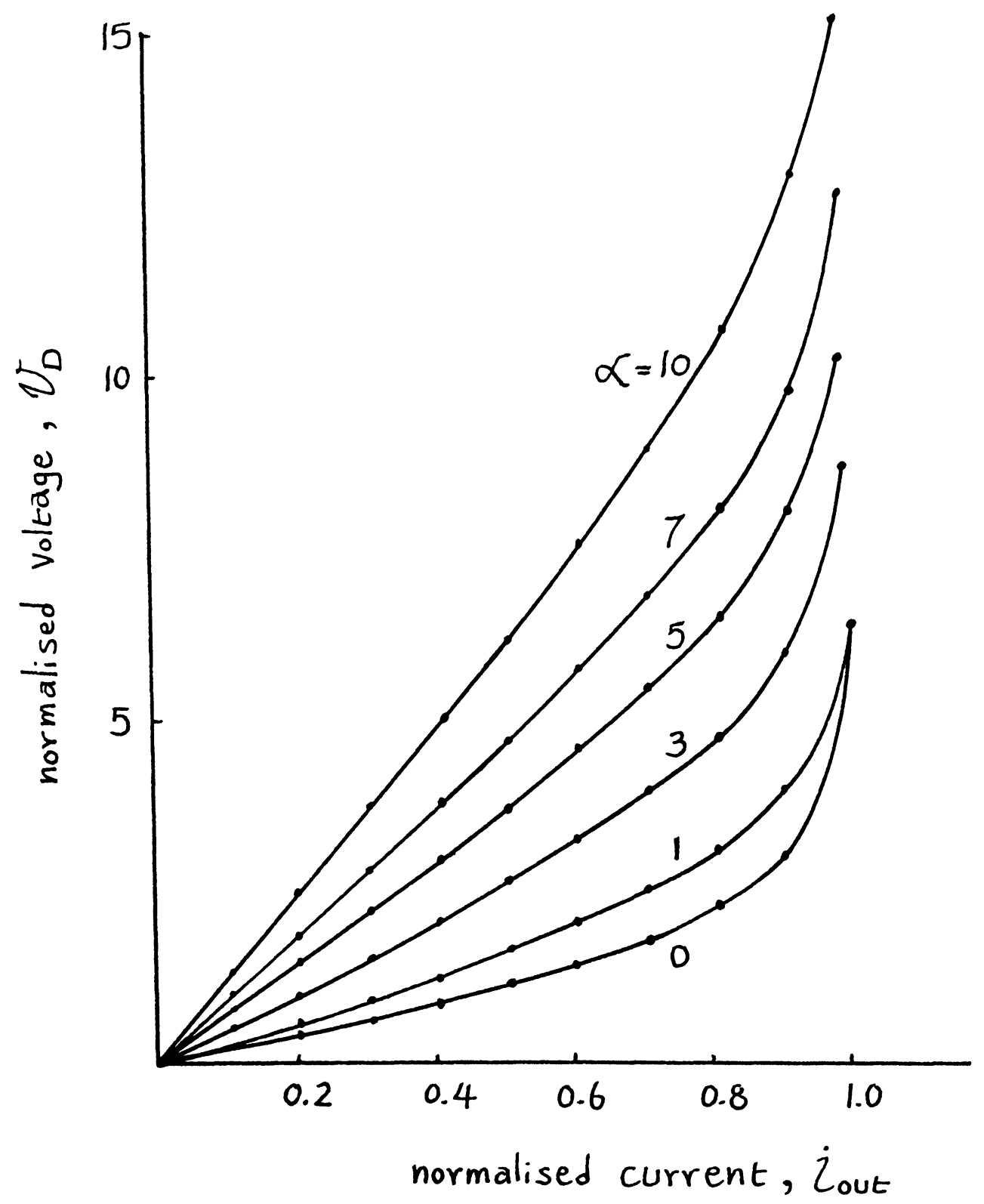

FIGURE 2 Current-voltage characteristic of the transconductance amplifier of Fig. 1

Substituting (15) into (1) yields

$$
\mathrm{i}_{\text {out }}=\sum_{\mathrm{n}=1.3 .5} \gamma_{\mathrm{n}} \sin \left(\frac{\mathrm{n} \pi}{2} \frac{\mathrm{t}}{\mathrm{T}}\right)
$$

From (15) and (16) we can see that an input voltage linearly changing with time will produce an output current sinusoidally changing with time, that is, the weak 
inversion MOSFET differential amplifier is working as a linear-to-sine function converter. However, the output sinusoidal current will contain third-harmonic and fifth-harmonic components. The total harmonic distortion can be expressed as

$\mathrm{THD}=\frac{\sqrt{\mathrm{I}_{3}^{2}+\mathrm{I}_{5}^{2}}}{\mathrm{I}_{1}}$

where $I_{m}, m=1,3,5$ is the amplitude of the mth-harmonic component of the output current. Using (7-9), (11-14), and (17), the variation of the THD with the parameter $\alpha$ was calculated and the results are shown in Fig. 3. From Fig. 3 we can see that the THD will be minimum for $\alpha \cong 4$. This result is slightly different from the observations of Tang et al. [2], which expects that the best performance of the weak-inversion MOSFET differential amplifier triangle-to-sinusoidal converter will be for $2<\alpha<3.33$. The results of Tang et al. [2] were obtained by

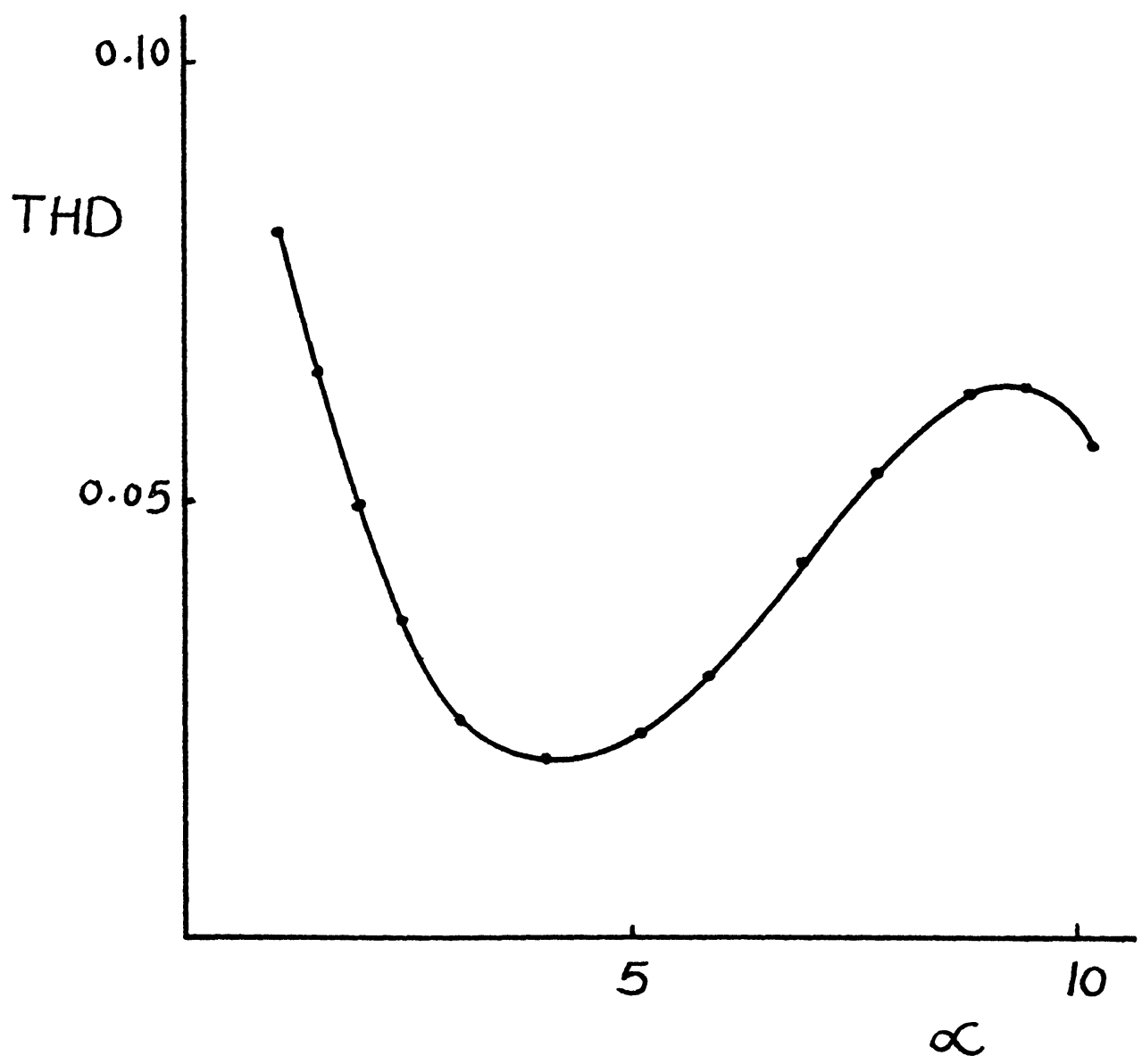

FIGURE 3 THD of the transconductance amplifier of Fig. 1 


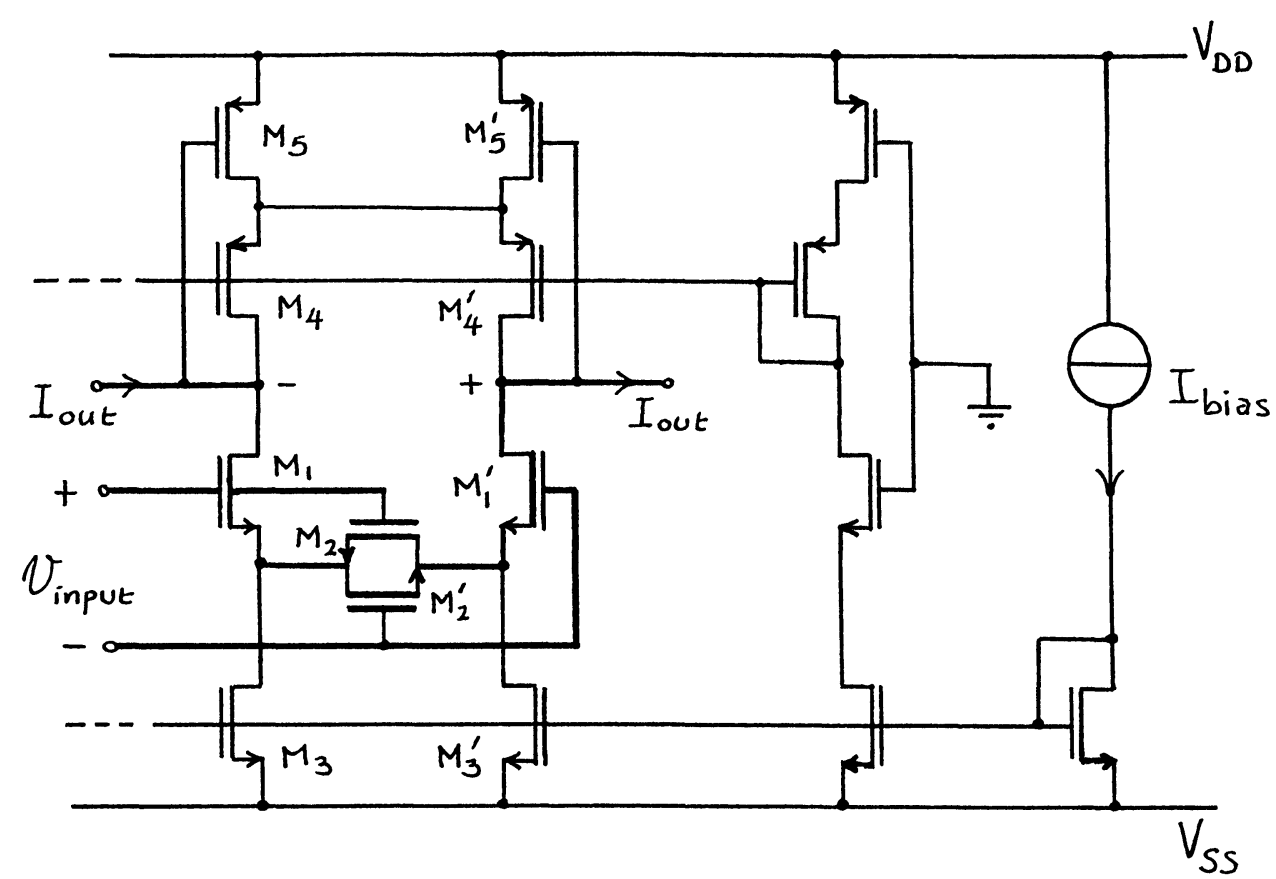

FIGURE 4 CMOS differential transconductance amplifier with the four-transistor core cell (in thick lines)

expanding (10) into a fifth-order polynomial. Obviously, this approximation, in contrast with that of $(1)$, is valid only for small values of $i_{\text {out }}$. This explains the difference between the results obtained using the present analysis and that reported by Tang et al. The present analysis is more accurate as it is valid over a wider range of input voltage and output currents.

\subsection{Example 2}

Consider the CMOS differential transconductance amplifier of Fig. 4. Assuming that the transistors $\mathbf{M}_{1}, \mathbf{M}_{1}^{\prime}, \mathbf{M}_{2}$ and $\mathbf{M}_{2}^{\prime}$ are in a common well and are working in the strong inversion region, it has been shown that the normalized current-voltage characteristic of the four-transistor core cell of Fig. 4 can be expressed by [3]

$$
\begin{aligned}
& \mathrm{i}_{\text {out }}=\nu_{\mathrm{D}} \sqrt{1-\nu_{\mathrm{D}}^{2} / 4} \\
& \text { for }\left|\nu_{\mathrm{D}}\right|<\sqrt{\frac{\mathrm{a}^{2}+\mathrm{a}+0.5}{\mathrm{a}^{4}+0.25}} \\
& \mathrm{i}_{\text {out }}= \pm \frac{\left(\mathrm{a} \nu_{\mathrm{D}} \sqrt{4 \mathrm{a}-2} \pm \sqrt{4 \mathrm{a}-1-\mathrm{a}^{2} \nu_{\text {out }}^{2}}\right)^{2}}{(4 a-1)^{2}}
\end{aligned}
$$


for

$\sqrt{\frac{\mathrm{a}^{2}+\mathrm{a}+0.5}{\mathrm{a}^{4}+0.25}}<\left|\nu_{\mathrm{D}}\right|<\frac{\sqrt{4 a-2}}{\mathrm{a}}$

and

$\mathrm{i}_{\text {out }}= \pm 1$

for $\left|\nu_{D}\right| \geqslant \frac{\sqrt{4 a-2}}{a}$

where

$$
\begin{aligned}
\mathrm{a} & =1+\alpha \\
\alpha & =\beta_{1} / 4 \beta_{2} \\
\nu_{\mathrm{D}} & =g_{\mathrm{mo}} \nu_{\text {input }} / \mathrm{I}_{\text {bias }} \\
\mathrm{i}_{\text {out }} & =\mathrm{I}_{\text {out }} / \mathrm{I}_{\text {bias }} \\
\mathrm{g}_{\mathrm{mo}} & =\frac{\mathrm{I}_{\text {bias }}}{\mathrm{a}\left(\mathrm{V}_{\mathrm{GS}}-\mathrm{V}_{\mathrm{T}}\right)_{\mathrm{M}_{\mathrm{l}}}}
\end{aligned}
$$

$\nu_{\text {input }}$ is the differential input voltage, $\mathrm{I}_{\text {out }}$ is the output current, $\beta_{\mathrm{i}}, \mathrm{i}=1,2$ is the transconductance constant of the MOSFET transistors where $\beta=\mu_{\mathrm{o}} \mathrm{C}_{\mathrm{ox}} \frac{\mathrm{W}}{\mathrm{L}}, \mu_{\mathrm{o}}$ is the mobility of carriers, $\mathrm{C}_{\mathrm{ox}}$ is the gate capacitance/unit area, $\mathrm{W}$ and $\mathrm{L}$ are the channel width and length respectively, $\mathrm{V}_{\mathrm{T}}$ is the threshold voltage of the MOSFET transistor, and $\left(\mathrm{V}_{\mathrm{GS}}-\mathrm{V}_{\mathrm{T}}\right)_{\mathrm{M}_{1}}$ is the quiescent gate overdrive voltage of the MOSFET $\mathrm{M}_{1}$.

Equation (18) in its present form cannot be used to investigate the non-linear performance of the CMOS differential transconductance amplifier of Fig. 4 as this requires a single expression that stretches over the whole region of operation.

Using the procedure described in the preceding sections, (18) can be approximated by the sine-series function of (1), with $x=\nu_{D}$ and $y=i_{\text {out }}$, for any value of the parameter $\alpha$. For different values of $\alpha$, the parameters B and $\gamma_{n}, n=1,3$, 5 were obtained and fitted to (2), giving

$$
\begin{aligned}
& \gamma_{1}=0.966-1.6384\left(\frac{\alpha}{100}\right)+5.218\left(\frac{\alpha}{100}\right)^{2}-8.797\left(\frac{\alpha}{100}\right)^{3} \\
&+ 7.301\left(\frac{\alpha}{100}\right)^{4}-2.3458\left(\frac{\alpha}{100}\right)^{5}
\end{aligned}
$$




$$
\begin{aligned}
100 \gamma_{3}=-2.792-116.667\left(\frac{\alpha}{100}\right)+ & 366.816\left(\frac{\alpha}{100}\right)^{2}-610.624\left(\frac{\alpha}{100}\right)^{3} \\
& +502.194\left(\frac{\alpha}{100}\right)^{4}-160.328\left(\frac{\alpha}{100}\right)^{5} \\
100 \gamma_{5}=0.6+47.173\left(\frac{\alpha}{100}\right)-154.993\left(\frac{\alpha}{100}\right)^{2}+269.1\left(\frac{\alpha}{100}\right)^{3} & -224.204\left(\frac{\alpha}{100}\right)^{4}+74.255\left(\frac{\alpha}{100}\right)^{5}
\end{aligned}
$$

and

$\mathrm{B}=2 \sqrt{2} \sqrt{\frac{1+2 \alpha}{1+\alpha}}$

Using (1) and (19-22), calculations were made and the results are shown in Fig. 5. From Fig. 5, it is obvious that the proposed approximation accurately represents the input-output characteristic of the CMOS differential transconductance amplifier of Fig. 4.

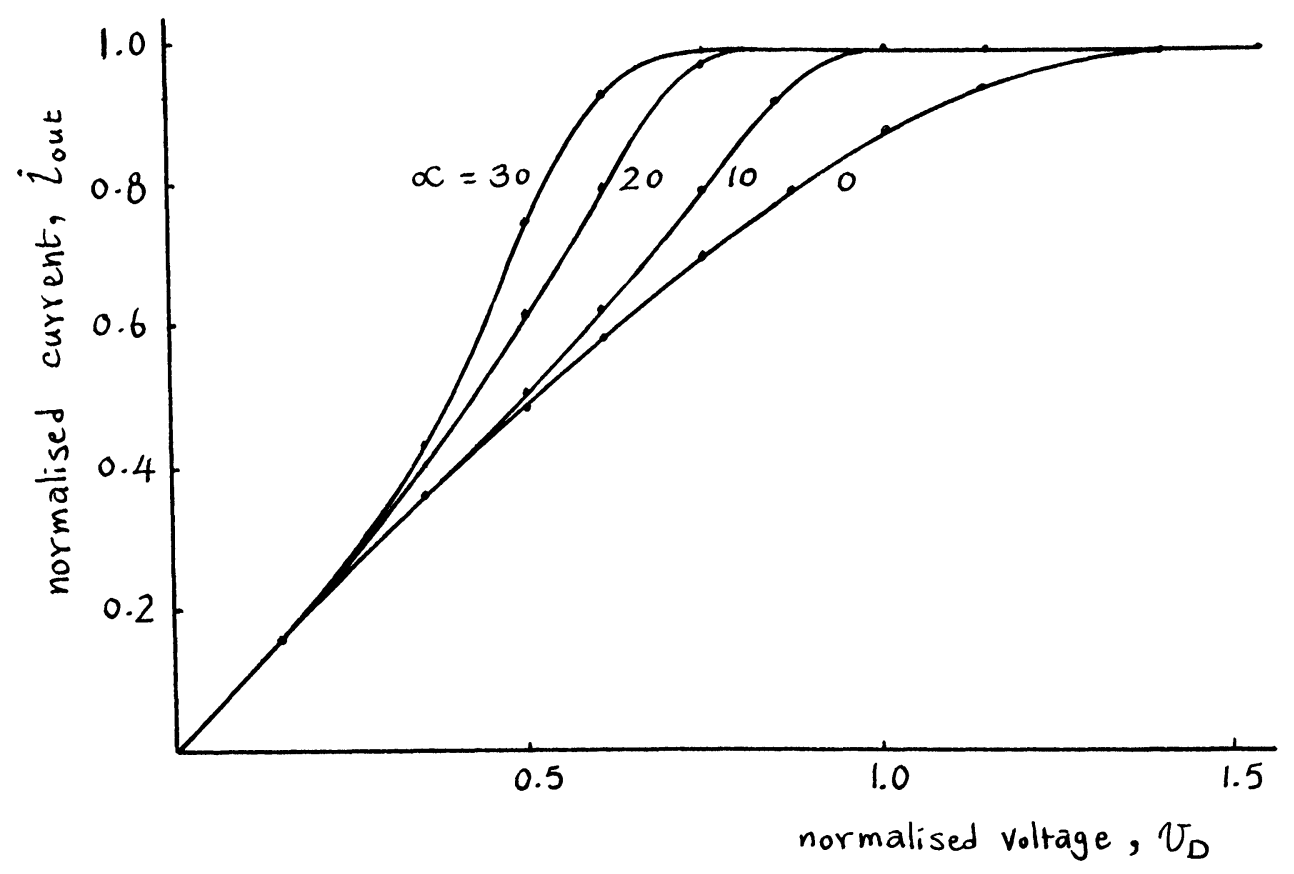

FIGURE 5 Normalised i-v characteristic of the four transistor core cell of Fig. 4 
Now, consider a CMOS differential transconductance amplifier excited by a single sinusoid of the form

$\nu_{\mathrm{D}}=\mathrm{V} \sin \omega \mathrm{t}$

where $\mathrm{V}=\mathrm{B} / 2$. Using (7-9), (19-22), and (17) the total harmonic distortion defined by (17) was calculated for different values of the parameter $\alpha$ and the results are shown in Fig. 6. From Fig. 6, we can see that the optimum value of $\alpha$ for best linearity performance, that is minimum THD, is 9.2 . This value is different from the 6.7 conjectured by Krummenacher et al. [3].

\subsection{Example 3}

Consider the source-coupled pair of Fig. 7. Assuming that the two transistors are working in the strong inversion, the current-voltage characteristic of the sourcecoupled pair can be expressed as [4]

$\mathrm{i}_{\mathrm{out}}=2 \nu_{\mathrm{D}} \sqrt{\alpha-\nu_{\mathrm{D}}^{2}}$

where $\mathrm{i}_{\text {out }}=\frac{\mathrm{I}_{\mathrm{out}}}{\beta / 4}, \nu_{\mathrm{D}}=\nu_{\text {input }}, \alpha=4 \frac{\mathrm{I}_{\mathrm{ss}}}{\beta}$ where $\beta$ is the transconductance constant of the MOSFET.

Equation (24) in its present form cannot be used to investigate the non-linear performance of the source-coupled MOS transconductance amplifier of Fig. 6. This

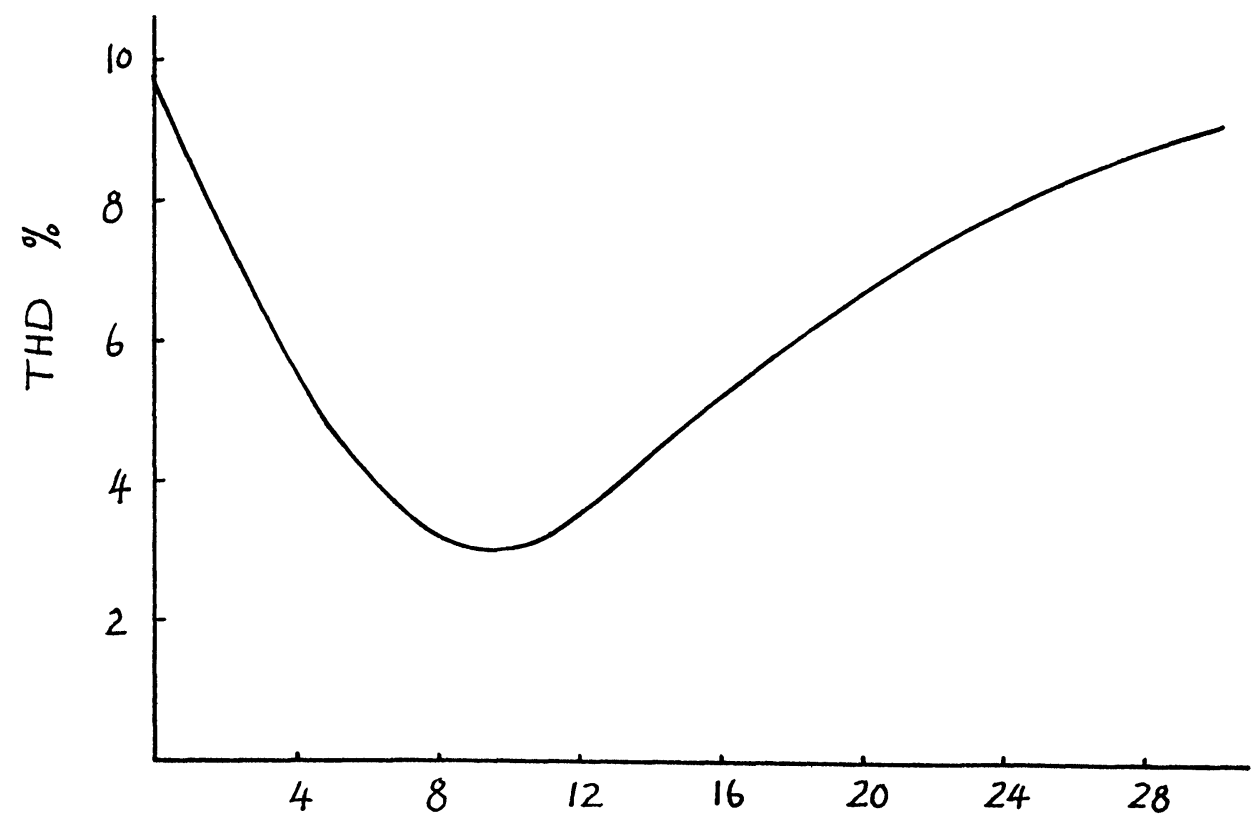

FIGURE 6 THD of the four-transistor core cell of Fig. 4 


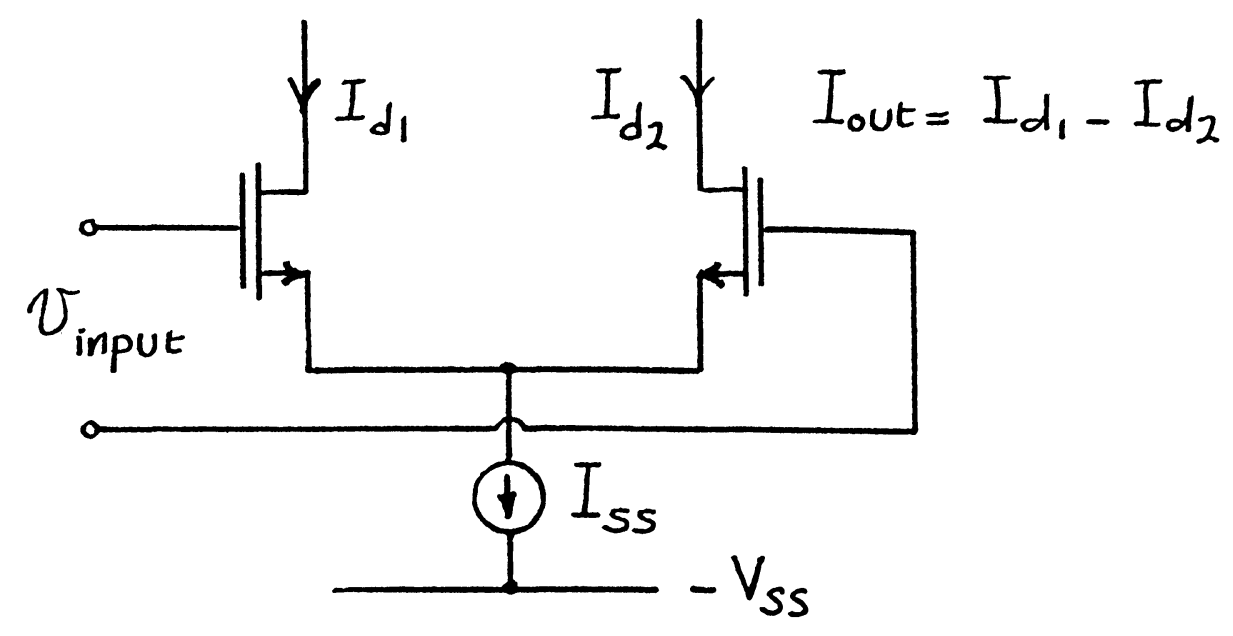

FIGURE 7 Source-coupled MOS transconductance amplifier

is attributed to the untractable square-root term. Using the procedure described in the preceding sections, (24) can be approximated by the sine-series function of (1), with $\mathrm{x}=\nu_{\mathrm{D}}$ and $\mathrm{y}=\mathrm{i}_{\text {out }}$ for any value of $\alpha$. For different values of $\alpha$, the parameters $B$ and $\gamma_{n}, n=1,3,5$ were obtained and fitted to (2), giving

$$
\begin{aligned}
\gamma_{1}= & 0.9619 \alpha \\
\gamma_{3}= & 0.036(1-\alpha) \\
\gamma_{5}=0.03+0.032\left(\frac{\alpha}{10}\right)-1.963\left(\frac{\alpha}{10}\right)^{2} & +7.9375\left(\frac{\alpha}{10}\right)^{3} \\
& -13.125\left(\frac{\alpha}{10}\right)^{4}+6.9375\left(\frac{\alpha}{10}\right)^{5}
\end{aligned}
$$

and

$\mathrm{B}=\sqrt{2 \alpha}$

Using (1) and (25-28), calculations were made and the results are shown in Fig. 8. From Fig. 8, it is obvious that the proposed approximation accurately represents the input-output characteristic of the source-coupled pair of Fig. 7.

Now, consider a source coupled pair excited by a single sinusoid of the form of (23). Using (7-9) and (25-28), the relative third-harmonic and fifth-harmonic distortions expressed by $I_{3} / I_{1}$ and $I_{5} / I_{1}$ were calculated and the results are shown in Fig. 9. From Fig. 9, we can see that, as expected from Fig. 8, the non-linear distortion decreases with the increase of the parameter $\alpha=4 \frac{I_{s s}}{\beta}$. This means that the range of input voltage over which the source-coupled transconductance amplifier behaves approximately as a linear component can be increased by increasing 


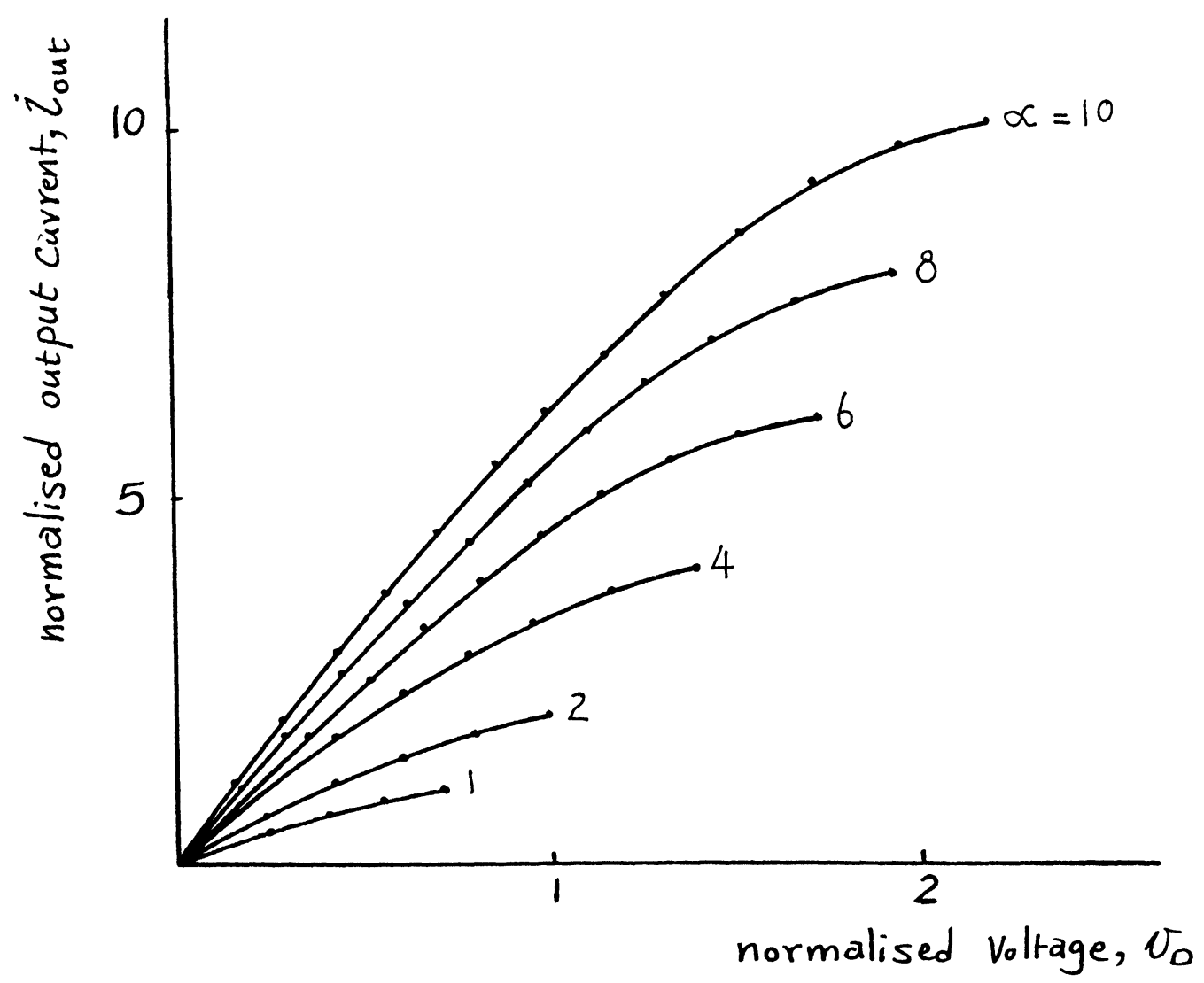

FIGURE 8 Input-output characteristic of the source-coupled amplifier of Fig. 7

the current $I_{s s}$ or decreasing the parameter $\beta$, which in-turn depends on the physical parameters and dimensions of the devices.

\section{CONCLUSION}

In this paper, a simple procedure has been presented for approximating the nonlinear input-output characteristics of electronic circuits. In principal, the non-linear characteristics is represented by a sine-series. The coefficients of this sine-series can be easily calculated using simple hand calculations. Using this sine-series approximation, the harmonic and intermodulation performance of non-linear electronic circuits can be predicted using closed-form analytical expressions. These expressions are simple and the Bessel functions involved can be computed either by built-in subroutines in the mainframe computers or by using trigonometric approximations for the Bessel functions [5, 6], which are especially convenient for personal computers and pocket calculators. The analytical expressions obtained in this paper can be used for quantitative study of the effect of the device parameters 


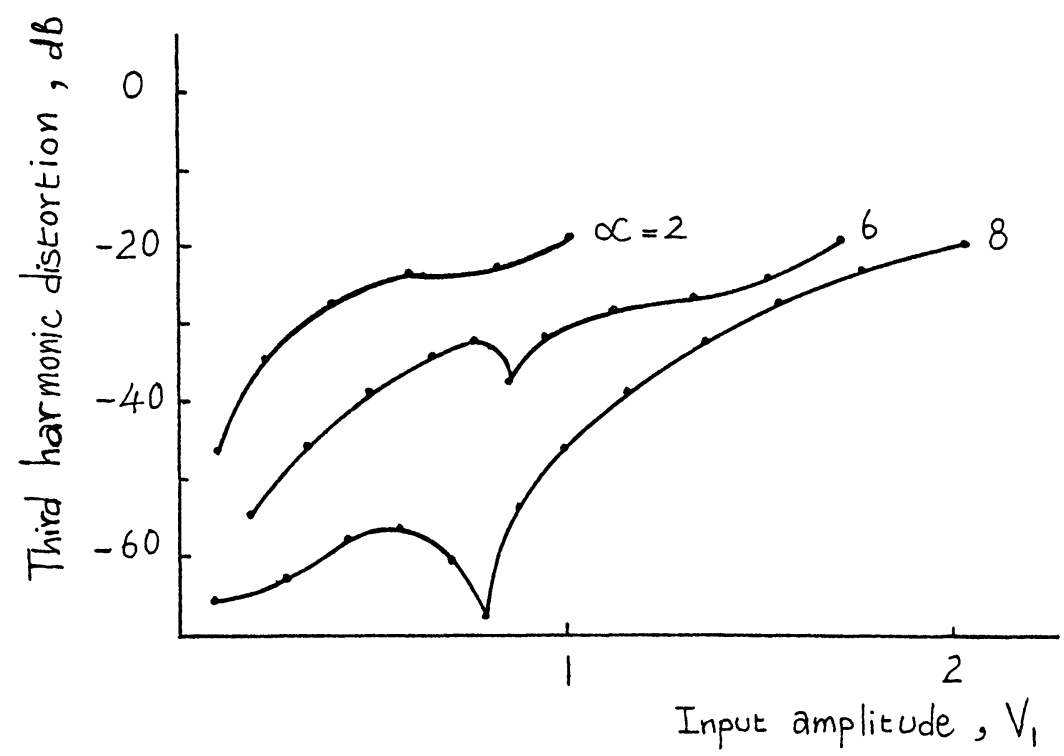

(2)

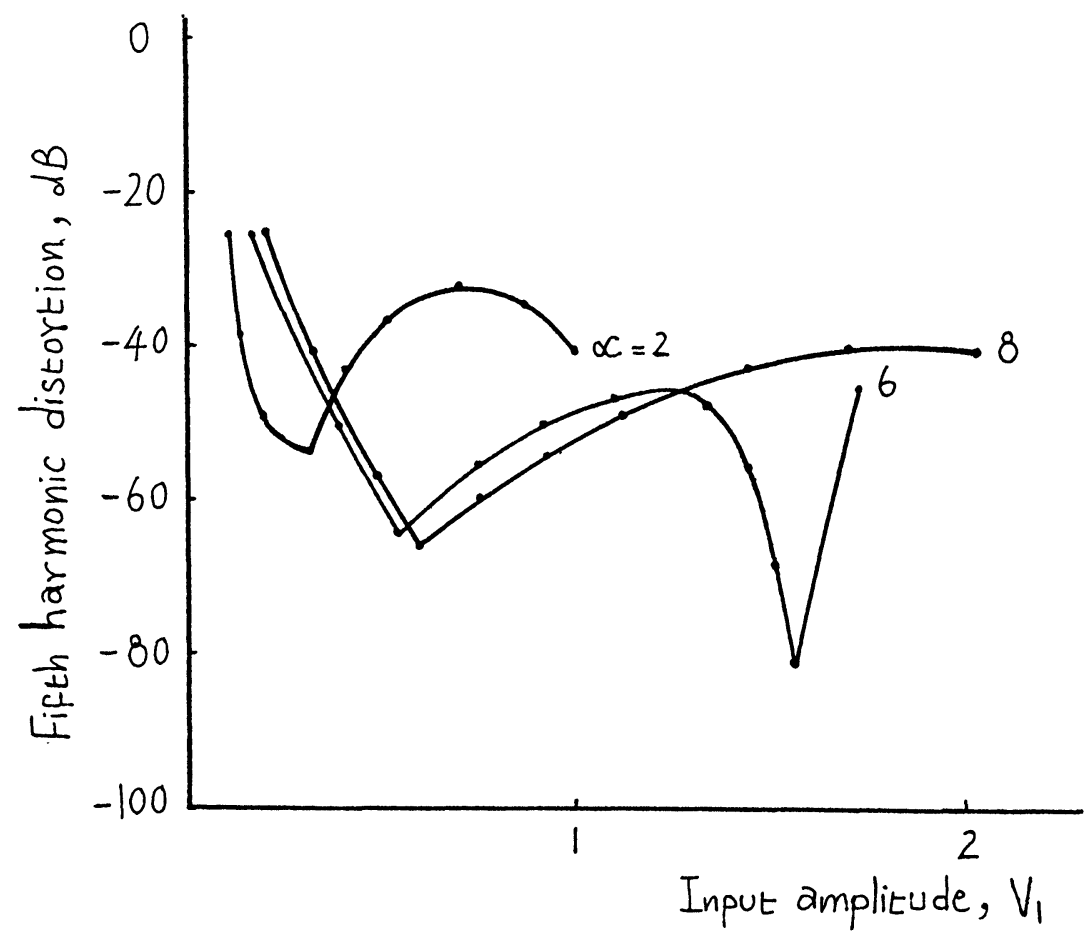

(b)

FIGURE 9 Variation of the (a) third-harmonic and (b) fifth-harmonic distortion of the transconductance amplifier of Fig. 7. 
on the performance of electronic circuits. In this regard, the harmonic distortion performance of three widely used transconductance amplifiers was considered. This can help in selecting the values of the MOSFET parameters, which will yield a prespecified linearity performance of the transconductance amplifiers.

\section{REFERENCES}

1. I.A. Dodes, Numerical analysis for computer science, Elsevier North Holland, New York, 1978

2. Z. Tang, $O$. Ishizuka and $H$. Matsumoto, MOS triangle-to-sine wave converter based on subthreshold operation, Electronics Letters, Vol. 26, 1990, pp. 1983-1985

3. F. Krummenacher and N. Joehl, A 4-MHz CMOS continuous-time filter with on-chip automatic tuning, IEEE Journal of Solid-State Circuits, Vol. 23, 1988, pp. 750-758

4. P.R. Gray and R.G. Meyer, Analysis and design of analog integrated circuits, John Wiley \& Sons, New York, 1984, Chapter 12.

5. N.M. Blachman and S.H. Mousavinezahd, Trigonometric approximations for Bessel functions, IEEE Transactions on Aerospace and Electronic Systems, Vol. AES-22, 1986, pp. 2-7

6. R.A. Waldron, Formulas for computation of approximate values of some Bessel functions, Proceedings IEEE, Vol. 69, 1981, pp. 1568-1588 

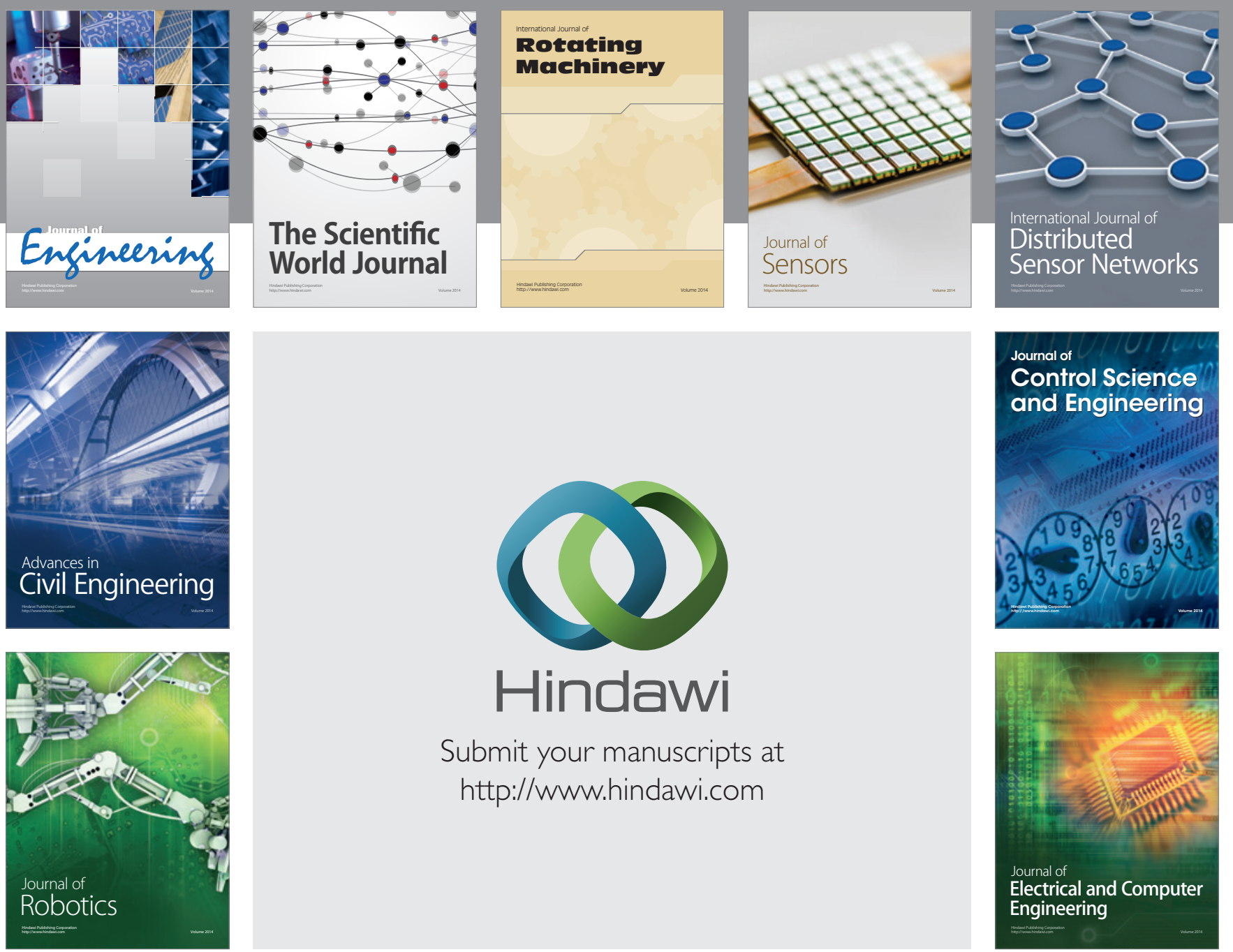

Submit your manuscripts at

http://www.hindawi.com
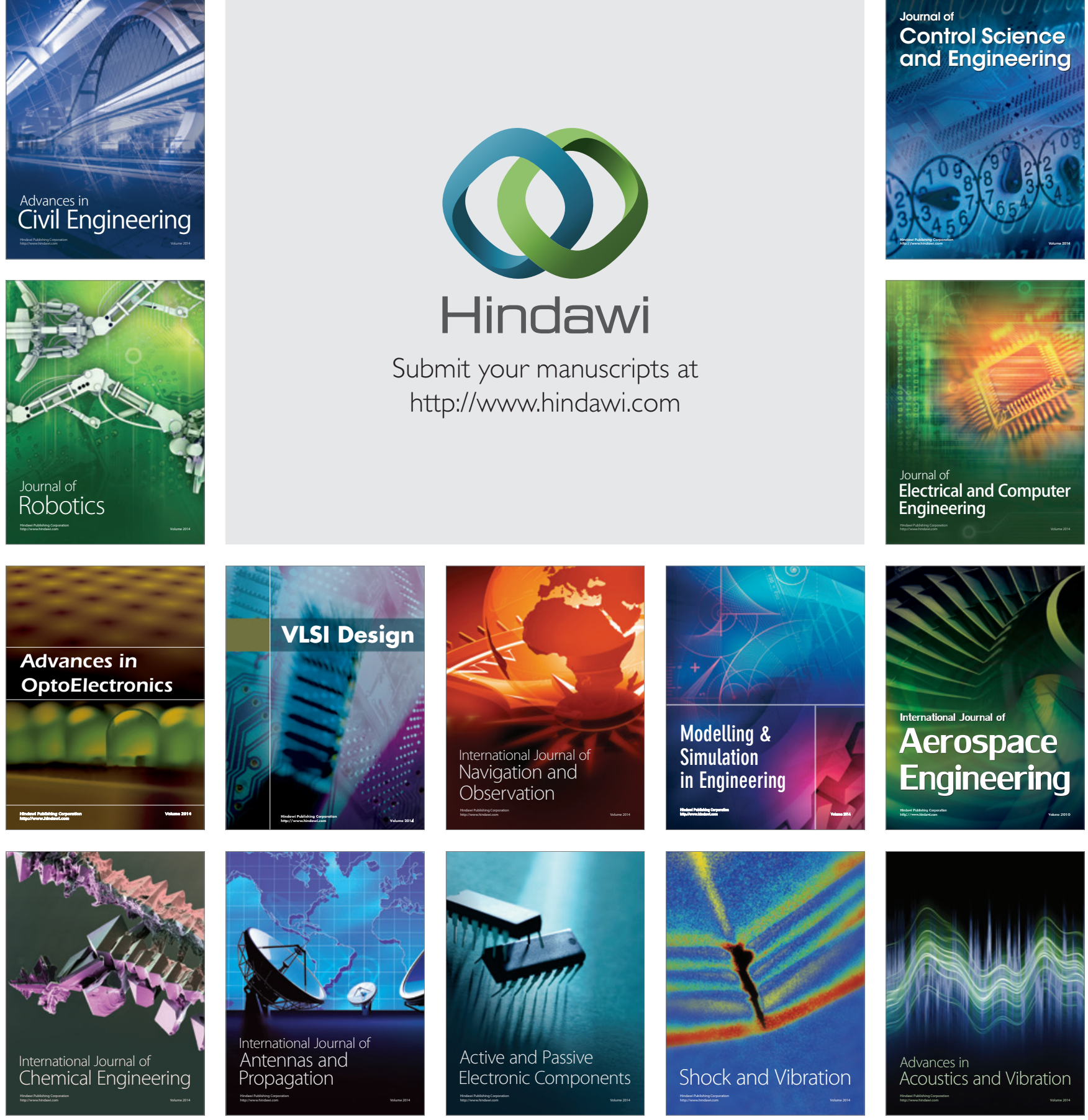